\title{
An Evaluation of Inter-Fractional Set-Up Errors in Patients Treated with Distinct Immobilization Equipment for Varying Anatomical Regions
}

\author{
Sibel Karaca, Hamit Başaran, Timur Koca, Ferhat Özbayrak \\ Department of Radiation Oncology, Erzurum Regional Training and Research Hospital, Erzurum, Turkey \\ Email: sibeltuzlaci@gmail.com
}

Received 9 March 2016; accepted 16 May 2016; published 19 May 2016

Copyright $(\subset 2016$ by authors and Scientific Research Publishing Inc.

This work is licensed under the Creative Commons Attribution International License (CC BY). http://creativecommons.org/licenses/by/4.0/

(c) (;) Open Access

\section{Abstract}

This study aims to evaluate inter-fractional set-up errors in patients treated with distinct immobilization equipment (thermoplastic mask, knee-fix and feet-fix, wing board and vac-lok) for four anatomical regions including brain, head and neck (HN), thorax and pelvis. Data of randomly selected 140 patients who were treated for four anatomical regions were obtained using Hi-Art Helical Tomotherapy (HT) system. Pre-treatment planning was based on automatic registration readings of computed tomography (CT) and mega-voltage computed tomography (MVCT) on a daily basis. Distinct immobilization equipment was used for varying anatomical regions. Individual mean set-up error $(M)$, systematic error $(\Sigma)$, and random error $(\sigma)$ values were calculated through daily translational and rotational deviation values. The size of translational, systematic and random error was 1.31 - $4.93 \mathrm{~mm}$ for brain, 2.28 - $4.88 \mathrm{~mm}$ for $\mathrm{HN}, 4.04-9.90 \mathrm{~mm}$ for thorax, and $6.34-14.68 \mathrm{~mm}$ for pelvis. Rotational values were as follows: $0.06^{\circ}-0.73^{\circ}$ for brain, $0.42^{\circ}-0.6^{\circ}$ for $\mathrm{HN}, 0.48^{\circ}-1.14^{\circ}$ for thorax and $0.65^{\circ}-1.05^{\circ}$ for pelvis. The highest translational, systematic and random error value was obtained from the pelvic regional. The highest standard and random error value in pitch and roll was produced in the rotational direction of the pelvis $\left(0.05^{\circ}\right.$ and $\left.0.71^{\circ}\right)$, while the highest error value in yaw was $\left(1.14^{\circ}\right)$ produced from thorax. Inter-fractional set-up errors were most commonly produced in the pelvis, followed by thorax. Our study results suggest that the highest systematic and random errors are found for thorax and pelvis. Distinct immobilization equipment was important in these results. Safety margins around the clinical target volume (CTV) are changeable for different anatomical regions. A future work could be developed to new equipment for immobilization because of the reduced margins CTV.

\section{Keywords}

Hi-Art Helical Tomotherapy, IGRT, Inter-Fractional Set-Up Errors, Rotational Variation, 


\section{Translational Variation}

\section{Introduction}

Radiation therapy (RT) is the treatment modality using beams of X-rays, electrons or protons to treat cancer cells. After surgery, RT is the next most important method of curing cancer. There are two major considerations in RT: 1) providing a more homogenous dose distribution for the tumor and its surrounding; 2) preserving more healthy tissues. Inappropriate positioning causes both reduced dose for the target volume and radiating to healthy tissues. Side effects and local injuries resulting from healthy tissue radiation are also associated with position errors [1] [2]. In addition, set-up errors are inevitable during RT. These errors can be divided into two groups, including random and systematic errors [3] [4]. Systematic errors are defined as the limit differences between the simulation position and treatment position [5]. Random errors, on the other hand, consisted of positional errors such as immobilization, respiration, anatomical changes and other related problems [6]. RT is a complex treatment modality which requires quality assurance. Currently, two-dimensional conventional RT has been replaced by three-dimensional conformal RT and intensity-modulated radiotherapy (IMRT). The latter offers a wide conformality for the dose distribution, reducing organs at risk (OAR) dose and toxicity [7]. Compared to conventional treatment modalities, geometric uncertainties are more critical, and also detection and reduction of set-up errors are more invaluable in IMRT [8]. However, set-up errors may convert these advantages to disadvantages [9]. Therefore, appropriate IMRT use is based on minimizing set-up errors to provide true safety margins around CTV [10].

With the introduction of technological improvements, image-guided radiotherapy (IGRT) system has been developed to reduce possible set-up errors and increase target localization [11]. This system can improve the ability to accurately deliver highly conformal dose distributions in applications where set-up uncertainty or organ motion is high [12]-[14]. Systems in use in people for image guidance include port films, electronic portal imaging, fluoroscopic imaging, ultrasound, implanted fiducial-based tracking systems, and helical and conebeam computed tomography [12] [15].

Furthermore, HT (Tomotherapy, Madison, WI) is a volumetric image-guided, intensity-modulated radiation therapy delivery system [16]. It exerts its effects at most when used in combination with on board MVCT, which provides set-up verification [17] [18]. This system offers an opportunity to tailor treatment and compare and match the MVCT images on a daily basis and CT scans. Therefore, set-up excellence is able to be obtained before treatment [19]-[21]. Moreover, HT system has three modes of rigid image registration: one based on bony anatomy, one based on soft tissue, or using both, depending on the threshold applied to the Hounsfield numbers [22]. It is able to determine the positional offsets in translational directions and rotation directions (left-right, anterior-posterior and superior-inferior, pitch, roll and yaw) [23]. Translational variations about lateral (x), longitudinal (y), vertical (z) and rotational variations about the superior-inferior SI axis (roll), anterior-posterior AP axis (yaw), and medial-lateral ML axis (pitch) are also recorded by the software. Roll variations are automatically corrected by the gantry at the time of treatment, whereas pitch and yaw corrections must be made manually. Pitch and yaw cannot be corrected because the couch does not move in this direction. However, Tomotherapy treats in a helical fashion, and roll can be adjusted [11] [24]. The MVCT images in the computer display of the treatment console during registration and CT images obtained from the CT simulator are overlapped using directional and rotational switches [24]. All patients are, then, treated when positioning is fully optimized. Thus, a number of set-up errors can be automatically detected and corrected by IGRT. In this study, we aimed to evaluate inter-fractional set-up errors in patients treated with distinct immobilization equipment for four anatomical regions, including brain, $\mathrm{HN}$, thorax, and pelvis using the automatic registration values of pre-treatment planning CT images and daily MVCT images.

\section{Materials and Methods}

Between 2011 and 2015, data of randomly selected 140 patients (57 brain, $27 \mathrm{HN}, 20$ thorax and 36 pelvis) who were treated for four anatomical regions were obtained using Hi-Art HT system. Before RT, CT images using $\mathrm{CT}$ simulation was obtained for treatment planning. Brain and HN patients were immobilized in supine position 
via three-clamp "head" and "head and neck" thermoplastic mask, thorax patients were immobilized in supine position with wing board; T-Grip and CIVCO "knee-fix and feet-fix" and pelvis patients were immobilized supine position with hip and pelvic Vac-Lok and CIVCO "knee-fix and "feet-fix". All patients were scanned with a 2 - 3 mm slice thickness in CT.

In Tomotherapy MVCT, the slice thickness options are $2 \mathrm{~mm}, 4 \mathrm{~mm}$, and $6 \mathrm{~mm}$ with "fine", "normal", and "coarse" modes. Normal acquisition mode was selected for HN and brain, while normal or coarse acquisition modes were used for thorax and pelvis. Duration for a gantry period was $10 \mathrm{sec}$ to obtain an image. The table speed for each gantry rotation was $4 \mathrm{~mm}$ in fine mode, $8 \mathrm{~mm}$ in normal mode, and $12 \mathrm{~mm}$ in coarse mode. The mean nominal amount of energy was 3.5 MV in the imaging mode. Imaging took 2 to 4 min. Scanned area was selected using the least PTV. Higher tumor volume required larger scanning.

An automatic "bone" and "bone and tissue" registration modes were used in all patients. Translational (lateral $(\mathrm{X})$, longitudinal $(\mathrm{Y})$, and vertical $(\mathrm{Z})$ ) and rotational (pitch, yaw, roll) deviation values were estimated by Tomotherapy software (Figure 1). Daily records of translational and rotational deviation values were utilized (Figure 2). Inter-fractional variations, which show daily set-up variations, include set-up alterations and changes based on organ movements. Statistical analysis was performed using automatic registration values of $140 \mathrm{pa}-$ tients to detect pre-treatment inter-fractional variations, excluding intra-fractional variations. Each patient had an individual treatment plan and fraction numbers. 2520 translational and rotational deviation values obtained from the baseline, mid-term, and end of the treatment were used considering the individual fraction values. Systematic errors and random errors were estimated. Calculations were based on the methods performed by Bijhold et al., Van Herk and Royal College of Radiologists. Statistical analysis was performed using ANOVA test with Tukey post-hoc methods, and multiple comparison studies (SPSS, v16.0, SPSS Inc., Chicago, IL, USA).

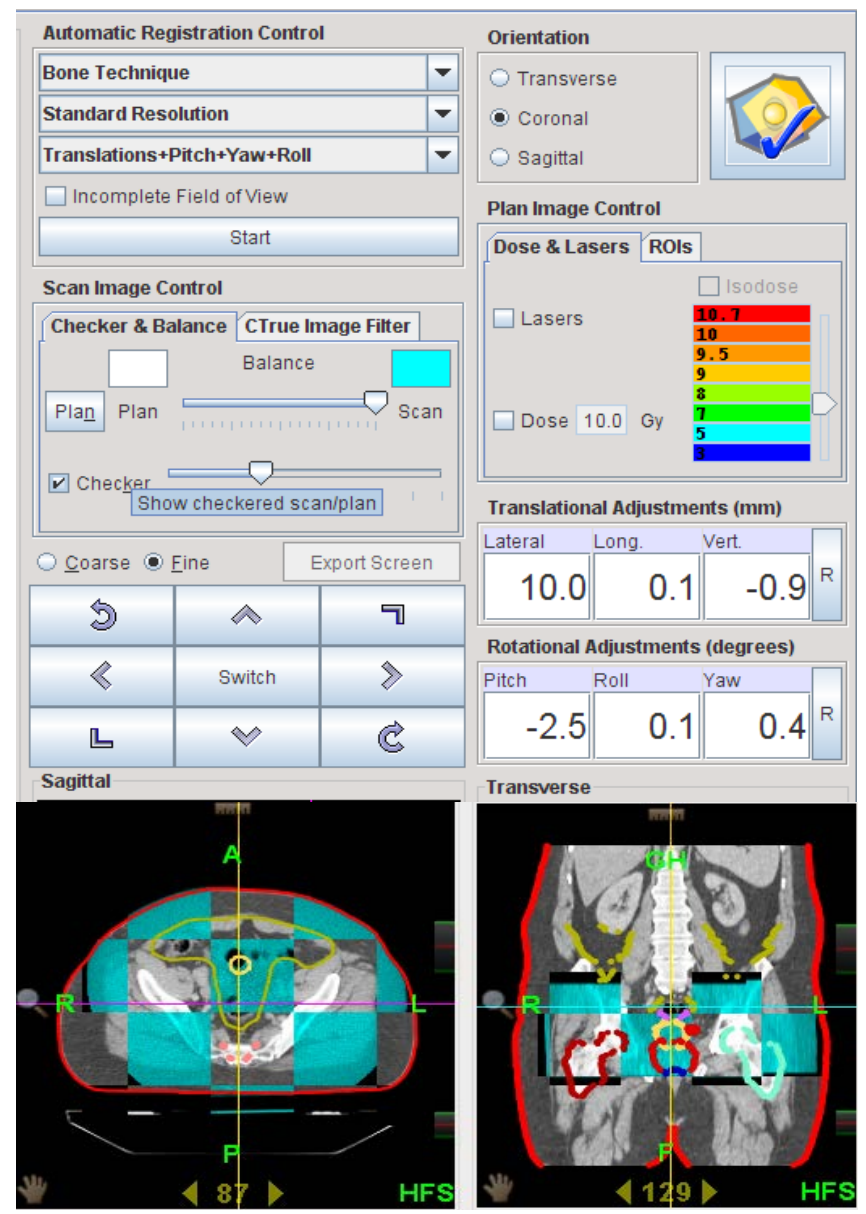

Figure 1. The registration panel showing the automatic and manual registration modes. Translational and rotational values are shown in display. 


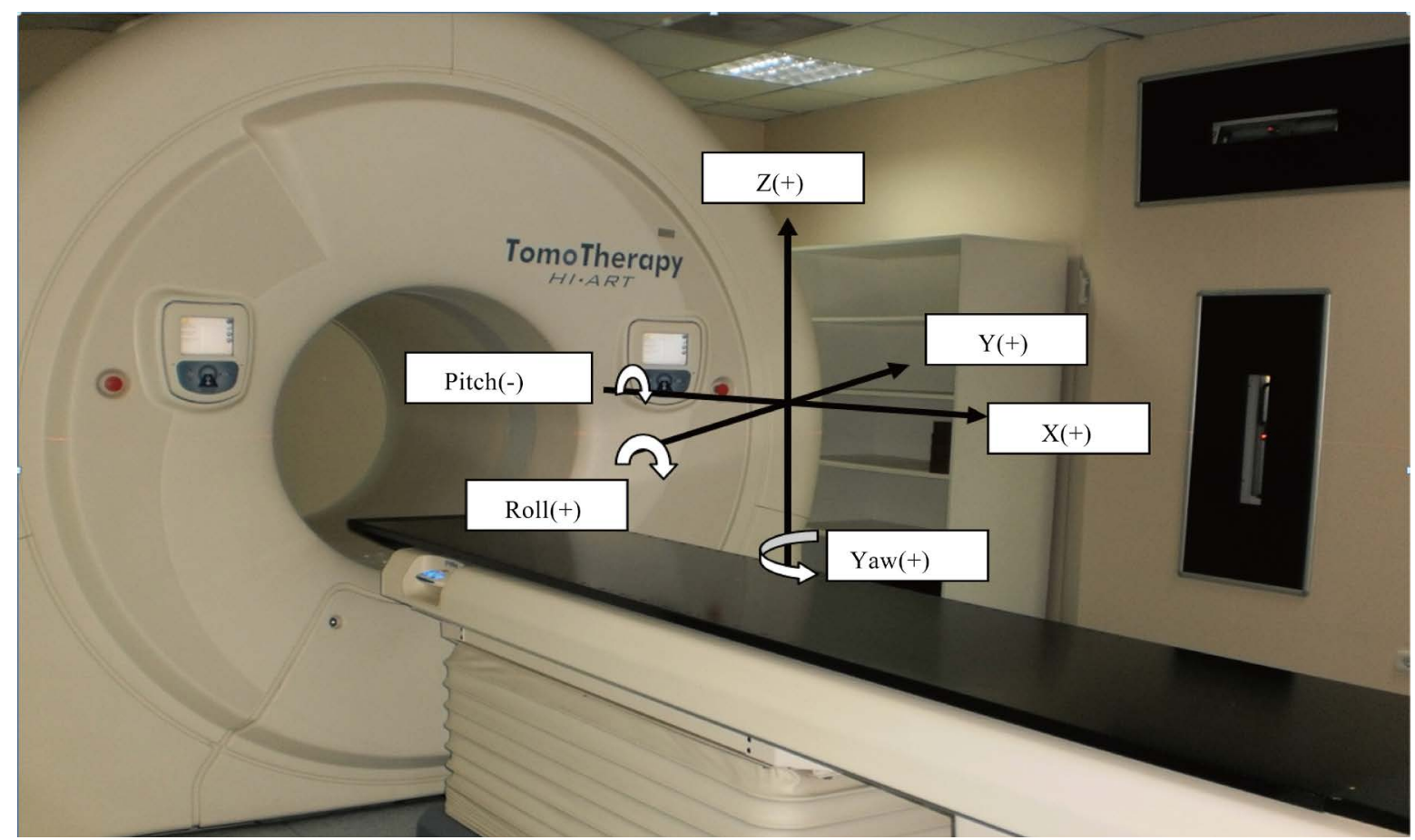

Figure 2. Diagram illustrating the positioning vectors of translational and rotational directions in Helical Tomotherapy system. Vectors of translational direction are lateral $(\mathrm{x})$, longitudinal $(\mathrm{y})$, vertical $(\mathrm{z})$. Pitch is an up-down rotation, yaw is a left-right rotation, and roll is a rotation along this line.

\section{Results}

Of 140 patients, translational and rotational deviation values for four anatomical regions are shown in Figure 3 and Figure 4. The patients were designated numbers based on the anatomical region ( 0 - 57 brain; 58 - $84 \mathrm{HN}$; 85 - 104 thorax; 105 - 140 pelvis). Table 1 shows the mean value $(\mathrm{M})$, standard deviation $(\Sigma)$, and random error $(\sigma)$ data of $\mathrm{X}, \mathrm{Y}, \mathrm{Z}$ directions in all anatomical groups. Table 2 shows the mean value $(\mathrm{M})$, standard deviation $(\Sigma)$, and random error $(\sigma)$ data of rotational pitch, roll, and yaw values. The major systematic error occurred in $\mathrm{X}$ and $Y$ directions in the pelvis $(7.35 \mathrm{~mm} ; 14.68 \mathrm{~mm})$, the highest standard error at $\mathrm{Z}$ direction was in thorax $(9.11$ $\mathrm{mm})$. Of three directions, the highest random error values were produced in the pelvis region $(6.33 \mathrm{~mm}, 12.54$ $\mathrm{mm}, 10.40 \mathrm{~mm}$, respectively). Systematic and random error values were similar in the translation directions of brain and $\mathrm{HN}$ and lower than of pelvis and thorax. In the rotational directions, however, the highest systematic error values for pitch, roll, and yaw were obtained from pelvis $\left(0.798^{\circ}, 0.65^{\circ}\right.$ and $0.988^{\circ}$, respectively). The highest random error values for pitch and roll were obtained from pelvis $\left(1.045^{\circ}, 0.714^{\circ}\right.$, respectively), whereas the highest random error value for yaw was produced from thorax $\left(1.14^{\circ}\right)$. A total of $99.8 \%$ of the rotational variation (pitch, roll, and yaw) values was $\pm 3^{\circ}$ in all anatomical regions. Figure 5 shows total systematic and random error for translational direction in all anatomical regions. Figure 6 illustrates total systematic and random error for rotational direction in all anatomical regions. Table 3 lists the inter-fractional set-up uncertainties for one site versus the rest using an ANOVA test with Tukey post-hoc methods. Statistical analysis revealed a very minor variation in the set-up alterations between brain and $\mathrm{HN}$, compared to thorax and pelvis. There was a statistically significant difference in the lateral $(\mathrm{X})$ direction between thorax and pelvis $(\mathrm{p}<0.05[0.044])$ and in the vertical $(X)$ direction between brain and thorax $(p<0.01[0.006])$, and thorax and pelvis $(p<0.05[0.023])$. In addition, there was a significant difference between brain and pelvis in the roll $(\mathrm{p}<0.01$ [0.0002]) and brain and thorax in yaw $(\mathrm{p}<0.01[0.0008])$. No significant difference in other directions was found.

\section{Discussion}

In the present study, we used automatic matching results of daily MVCT and planning CT images. These results were used to evaluate the inter-fractional set-up variations among the patients. We found similar but lower sys- 


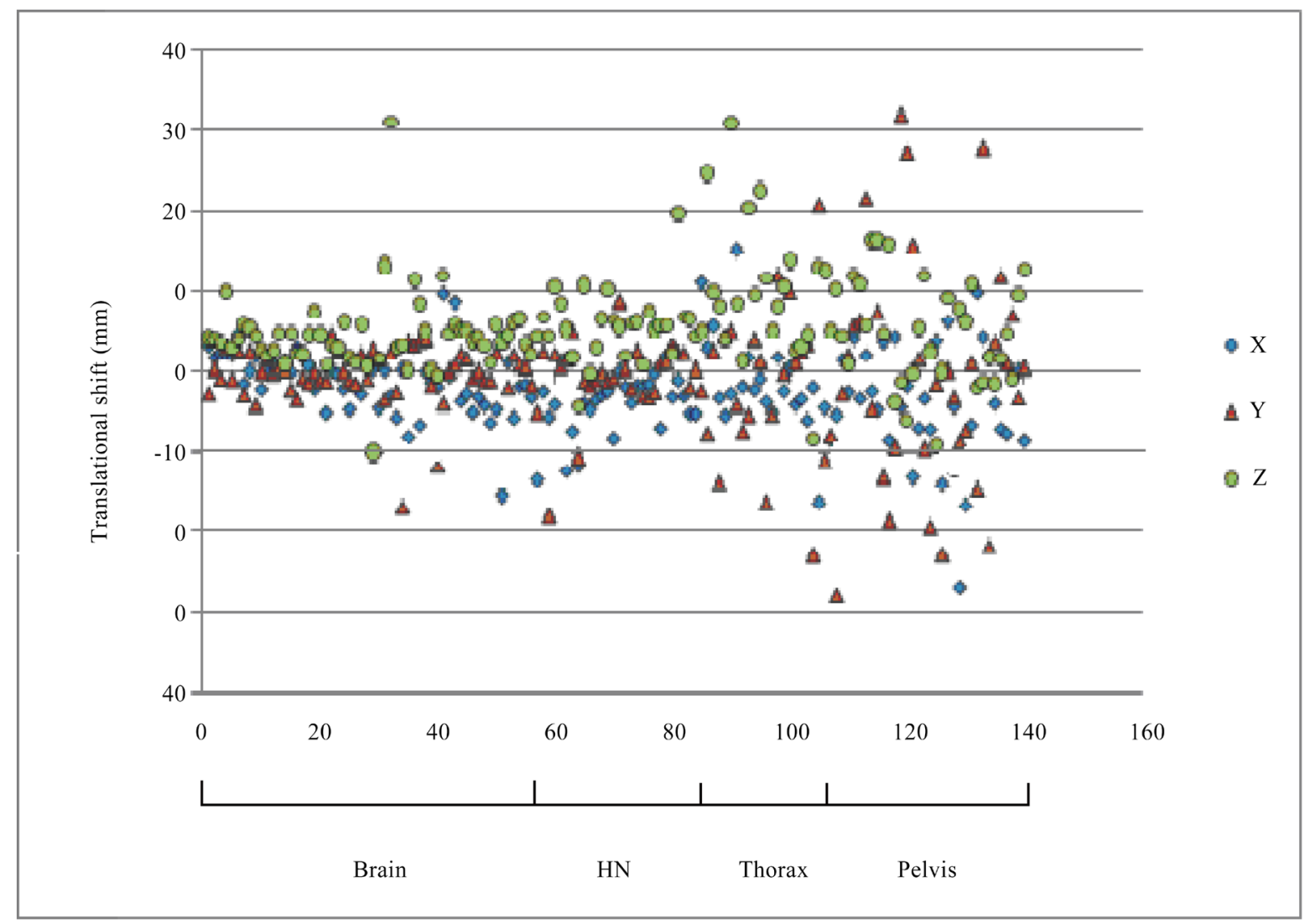

HN: Head and neck.

Figure 3. Translational deviation values for four anatomical regions on a daily MVCT/KVCT registration basis.

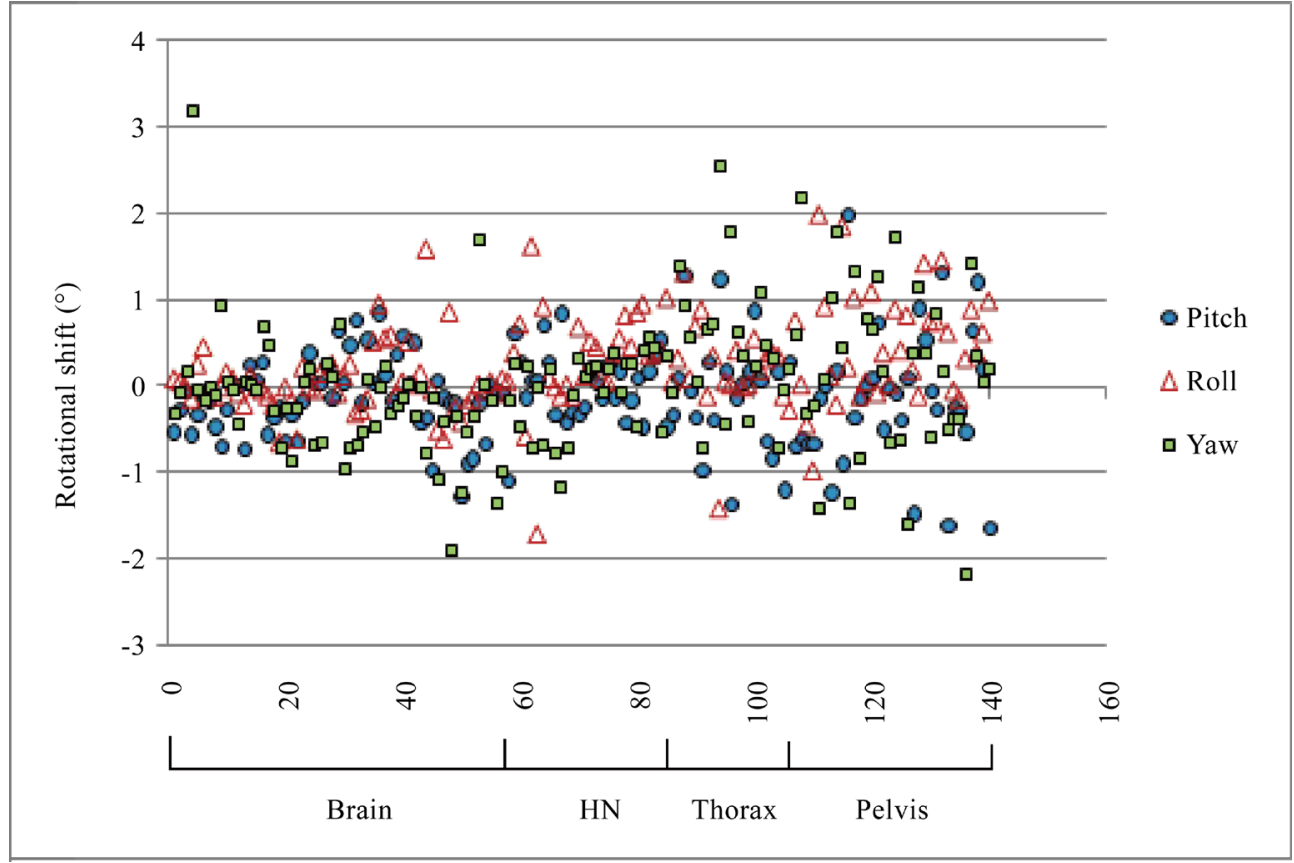

HN: Head and neck.

Figure 4. Rotational deviation values for four anatomical regions on a daily MVCT/KVCT registration basis. 
Table 1. The mean value $(\mathrm{M})$, standard deviation $(\Sigma)$, and random error $(\sigma)$ data of $\mathrm{X}, \mathrm{Y}, \mathrm{Z}$ directions in all anatomical groups.

\begin{tabular}{|c|c|c|c|c|c|c|c|c|c|c|}
\hline \multirow{2}{*}{ Site } & \multirow{2}{*}{$\begin{array}{c}\text { \# of } \\
\text { patients }\end{array}$} & \multicolumn{9}{|c|}{$X(\mathrm{~mm}) \mathrm{Y}(\mathrm{mm}) \mathrm{Z}(\mathrm{mm})$} \\
\hline & & M & $\Sigma$ & $\sigma$ & M & $\Sigma$ & $\sigma$ & $\mathbf{M}$ & $\Sigma$ & $\sigma$ \\
\hline Brain & 57 & -1.21 & 4.25 & 1.31 & -0.24 & 3.59 & 3.69 & 4.54 & 4.93 & 2.24 \\
\hline HN & 27 & -3.65 & 3.46 & 2.36 & -0.39 & 4.88 & 3.03 & 5.79 & 4.36 & 2.21 \\
\hline Thorax & 20 & -0.11 & 5.45 & 4.04 & -1.89 & 8.73 & 9.89 & 9.90 & 9.91 & 6.28 \\
\hline Pelvis & 36 & -4.00 & 7.35 & 6.34 & -0.31 & 14.68 & 12.54 & 4.91 & 7.17 & 10.40 \\
\hline
\end{tabular}

HN: Head and neck.

Table 2. The mean value (M), standard deviation $(\Sigma)$, and random error $(\sigma)$ data of rotational pitch, roll, and yaw values in all anatomical groups.

\begin{tabular}{|c|c|c|c|c|c|c|c|c|c|c|}
\hline \multirow{2}{*}{ Site } & \multirow{2}{*}{$\begin{array}{c}\text { \# of } \\
\text { patients }\end{array}$} & \multicolumn{9}{|c|}{$\operatorname{Pitch}\left({ }^{\circ}\right) \operatorname{Roll}\left({ }^{\circ}\right)$ Yaw( $\left({ }^{\circ}\right)$} \\
\hline & & M & $\Sigma$ & $\sigma$ & M & $\Sigma$ & $\sigma$ & M & $\Sigma$ & $\sigma$ \\
\hline Brain & 57 & -0.14 & 0.44 & 0.56 & -0.06 & 0.38 & 0.49 & 0.16 & 0.73 & 0.55 \\
\hline HN & 27 & -0.01 & 0.42 & 0.59 & 0.32 & 0.59 & 0.57 & -0.09 & 0.46 & 0.55 \\
\hline Thorax & 20 & -0.06 & 0.67 & 0.59 & 0.29 & 0.55 & 0.48 & 0.50 & 0.81 & 1.14 \\
\hline Pelvis & 36 & -0.16 & 0.79 & 1.04 & 0.47 & 0.65 & 0.71 & 0.18 & 0.99 & 0.92 \\
\hline
\end{tabular}

HN: Head and neck.

Table 3. Significance $(\mathrm{p}<0.05)$ of site-dependent inter-fractional set-up errors.

\begin{tabular}{|c|c|c|c|c|c|c|}
\hline & \multicolumn{3}{|c|}{ Brain } & \multicolumn{2}{|c|}{ HN } & \multirow{2}{*}{$\begin{array}{c}\text { Thorax } \\
\text { Pelvis }\end{array}$} \\
\hline & \multicolumn{2}{|c|}{ HN Thorax } & Pelvis & Thorax & Pelvis & \\
\hline Lateral(x) & NS & NS & NS & NS & NS & $\mathrm{p}<0.05$ \\
\hline Longitudinal (y) & NS & NS & NS & NS & NS & NS \\
\hline Vertical (z) & $\mathrm{NS}$ & $\mathrm{p}<0.05$ & NS & NS & NS & $\mathrm{p}<0.05$ \\
\hline Pitch & $\mathrm{NS}$ & NS & NS & NS & NS & NS \\
\hline Roll & $\mathrm{NS}$ & NS & $\mathrm{p}<0.05$ & NS & NS & NS \\
\hline Yaw & NS & $\mathrm{p}<0.05$ & NS & NS & NS & NS \\
\hline
\end{tabular}

NS: non-significant. HN: Head and neck.

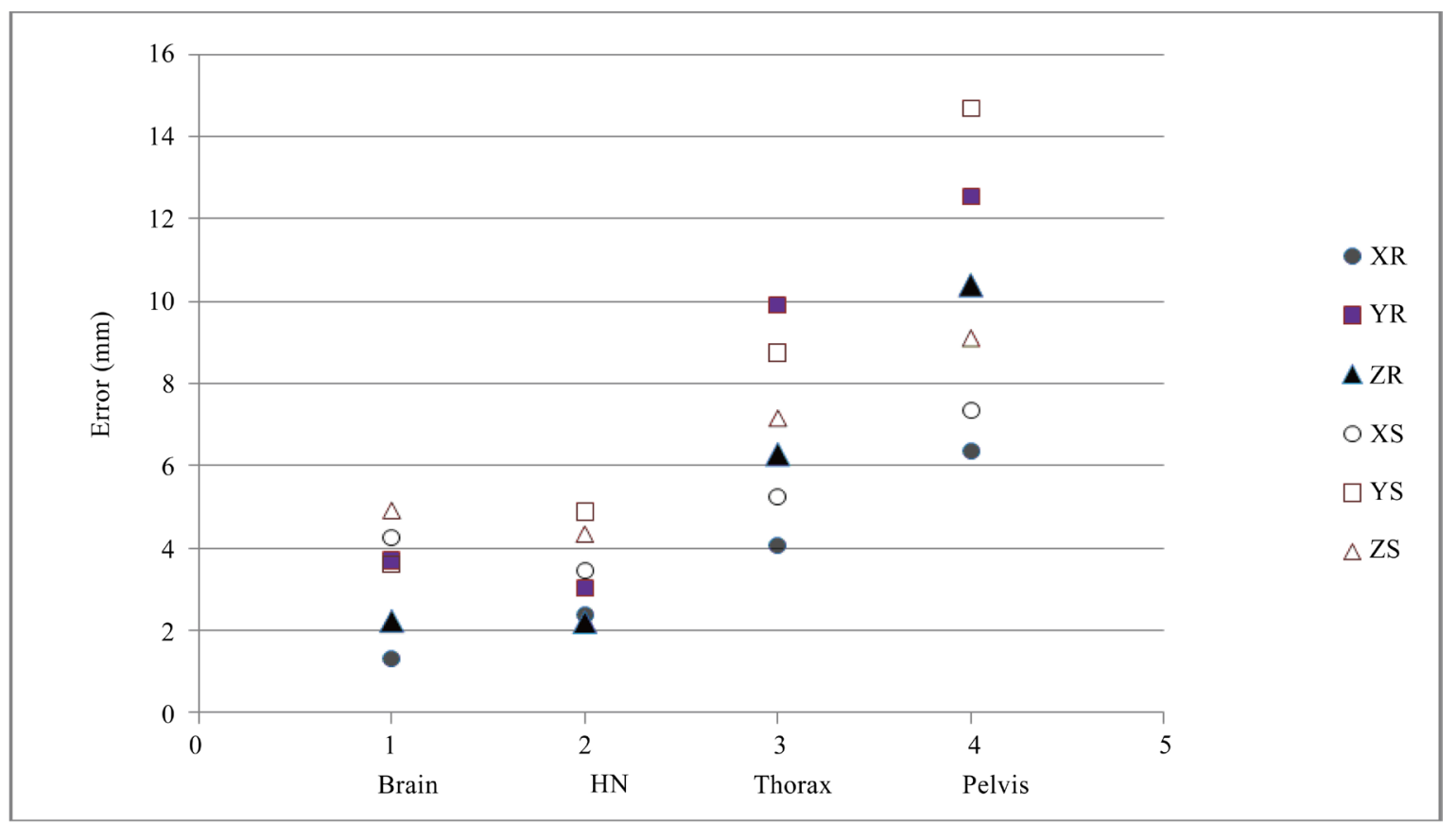

HN: Head and neck.

Figure 5. Total random (R) and systemic (S) errors for translational direction in all anatomical groups. 


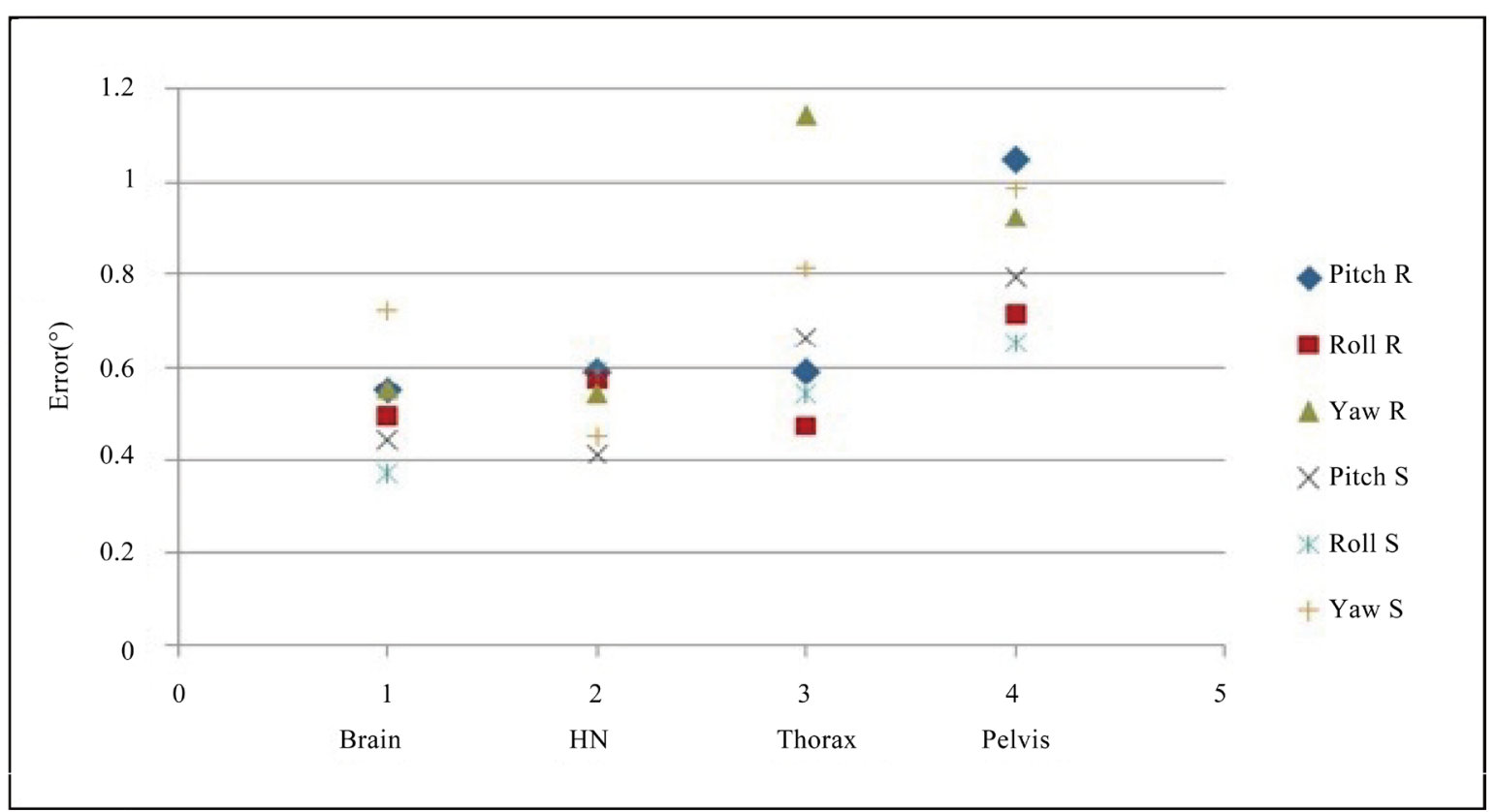

HN: Head and neck.

Figure 6. Total random (R) and systemic (S) errors for rotational direction in all anatomical groups.

tematic and random error values of brain and HN than thorax and pelvis. Similarly, Leah et al. reported lower systematic and random error values in the translational directions for brain and HN than for thorax and pelvis. There are several studies reporting lower set-up variation values for brain and HN compared to other anatomical regions [2] [12] [16] [28]. Brain and HN patients were immobilized in supine position via three-clamp 'head' and 'head and neck' thermoplastic mask. Thermoplastic masks and rigid anatomy offer a good immobilization; however. they are unable to fully eliminate translational and rotational errors [12]. Neck movements during set-up as well as inappropriate pillow use may result in such errors.

Although immobilization is of utmost importance for pelvis and thorax, some factors increase the translational and rotational error rates. In particular, major systematic and random errors, inappropriate positioning, careless handling of immobilization set on to the table and couch sag for overweight patients in the head feed position due to the lower localization of tumor may increase such errors. Another reason for increased set-up errors for pelvis is the full or empty status of rectum and bladder [12] [29]. In our study, inter-fractional set-up errors were most commonly produced in the pelvis, followed by thorax. Zhau et al. demonstrated that abdominal and pulmonary set-up uncertainties were more common than $\mathrm{HN}$ and brain probably due to the respiratory cycle. In addition, there are several studies showing that the highest set-up variation rate was located in the lungs due to the respiratory cycle [16]. Furthermore, wing board, an immobilization tool used for thoracic irradiation. as well as position errors in the T-Grip may lead to an increased set-up error rate. In this present study, we observed higher systematic and random error rates in the $\mathrm{Z}$ direction for thorax.

Despite higher translational systematic and random error rates in our study, we showed lower rotational systematic and random errors. Similarly, some studies reported lower rotational error rates [11] [12] [28]. A total of $99.8 \%$ of the rotational variation (pitch, roll, and yaw) values was $\pm 3^{\circ}$ in all anatomical regions. The rate of rotational variation was $94 \%$ in the study carried out by Kaiser and Schulthesis.

According to the position of the patient during simulation, different movements are typically present in the machine and these movements can be repeated every time. In addition, immobilization tools should be appropriately utilized to reduce intra-fractional variations during therapy [30]. The technician should work carefully throughout the treatment and the patient should be successfully immobilized to minimize random errors [11]. However, alterations in the irradiated area during the respiratory cycle and physical appearance (i.e. weight loss or gain), which are deemed as random errors, cannot be excluded. Such alterations are insignificant when they are within the range of tolerance. Furthermore, systematic errors should be minimized. Such errors can be reduced by checking the simulation and therapy tools on a daily, weekly, monthly and annually basis. Also, sys- 
tematic and random error rates may be increased by using different modes and lengths of MVCT imaging on different time points.

Furthermore, systematic and random error rates may play an important role in determining a safety CTV margin in RT planning. It is evident that possible set-up errors are considerably critical in the treatment with a low tumor range, such as IMRT. Therefore, possible causes of systematic and random errors should be identified and minimized. With the advent technology, IGRT system can reduce set-up errors and offer safer treatment of choice for the patients.

\section{Conclusion}

In conclusion, systematic and random set-up errors were most commonly produced in the pelvis, followed by thorax. We also found similar but lower systematic and random error values of brain and HN than thorax and pelvis. Our study results suggest that set-up error values vary in patients treated with distinct immobilization equipment for varying anatomical regions. Using various immobilization devices in different anatomical structures appears to influence on varying set-up error rates. In spite of modern techniques in RT, it is still impossible to reduce set-up errors to zero. Safety margins around the CTV are changeable for different anatomical region. CTV margins for these different anatomical groups in planning process must be evaluated more detailedly. A future work could be developed to new equipment for immobilization because of the reduced margins CTV.

\section{References}

[1] He, W., Vazquez, L.A., Dzintars, E., Papanikolaou, N. and Shi, C. (2010) Assessing the Dosimetric Consequence of Inter-Fractional Setup Shifts on Helical Tomotherapy Plans with Independent Dose Calculation. Journal of Cancer Science and Therapy, 2, 136-144. http://dx.doi.org/10.4172/1948-5956.1000039

[2] Chmielewski, L., Kukolowicz, P.F., Gut, P. and Debrowski, A. (2002) Assessment of the Quality of Radiotherapy with the Use of Portal and Simulation Images the Method and the Software. Journal of Medical Informatics and Technologies, 3, 171-179.

[3] Thasanthan, L., Piyasena, W.R.O., Croos, A.M.C., Dhanushia, R. and Narayanan, P.P. (2014) Assessment of ThreeDimensional Set-Up Errors in Pelvic Radiation Therapy. Journal of Cancer Prevention \& Current Research, 1, Article ID: 00009 .

[4] Gonzalez, J., Lopez, J., Azinovic, I., et al. (2013) Systematic and Random Patient Set-Up Errors in a Single-Radiation Center. Reports of Practical Oncology and Radiotherapy, 18, 303-398. http://dx.doi.org/10.1016/j.rpor.2013.03.398

[5] Mcgea, K.P., Dougles, M.S., Fein, A., et al. (1997) The Value of Set-Up Portal Films as an Estimate of a Patient's Position Throughout Fractionated Tangential Breast Irradiation: An On-line Study. Radiotherapy Oncology, 37, 223-228.

[6] Kukolowicz, P.F., Debrowski, A., Chmielewski, L., et al. (2005) Evaluation of Set-Up Deviations during the Irradiation of Patients Suffering from Breast Cancer Treated with Two Different Techniques. Radiotherapy Oncology, 75, $22-$ 27. http://dx.doi.org/10.1016/j.radonc.2005.02.004

[7] Sheng, K., Chow, J., Hunter, G., et al. (2008) Is Daily Computed Tomography Image Guidance Necessary for Nasal Cavity and Nasopharyngeal Radiotherapy? An Investigation Based on Helical Tomotherapy. The Journal of Applied Clinical Medical Physics, 9, 2686.

[8] Pehlivan, B., Pichenot, C. and Castaing, M. (2009) Inter-Fractional Set-Up Errors Evaluation by Daily Electronic Portal Imaging of IMRT in Head and Neck Cancer Patients. Acta Oncologica, 48, 440-445. http://dx.doi.org/10.1080/02841860802400610

[9] Suh, S. and Schultheiss, T.E. (2013) Distance-to-Agreement Investigation of Tomotherapy’s Bony Anatomy-Based Autoregistration and Planning Target Volume Contour-Based Optimization. International Journal of Radiation Oncology Biology Physics, 85, 862-865. http://dx.doi.org/10.1016/j.ijrobp.2012.06.015

[10] Fang, J.L., Guo, R., Yu, X., et al. (2013) Evaluation of Inter-Fraction and Intra-Fraction Errors during Volumetric Modulated Arc Therapy in Nasopharyngeal Carcinoma. Radiotherapy Oncology, 8, 78.

[11] Kaiser, A. and Schultheiss, T.E. (2005) Pitch. Roll and Yaw Variations in Patient Positioning. International Journal of Radiation Oncology Biology Physics, 66, 949-955. http://dx.doi.org/10.1016/j.ijrobp.2006.05.055

[12] Schubert, L.K., Westerly, D.C., Wolfgang, A.T., et al. (2009) A Comprehensive Assessment by Tumor Site of Patient Setup Using Daily MVCT Imaging from Over Three Thousand Eight Hundred Helical Tomotherapy Treatments. International Journal of Radiation Oncology Biology Physics, 73, 1260-1269. http://dx.doi.org/10.1016/j.ijrobp.2008.11.054

[13] Westerly, D.C., Soisson, E., Chen, Q., et al. (2009) Treatment Planning to Improve Delivery Accuracy and Patient 
Throughput in Helical Tomotherapy. International Journal of Radiation Oncology Biology Physics, 74, 1290-1297. http://dx.doi.org/10.1016/j.ijrobp.2009.02.004

[14] Welsh, J.S., Bradley, K., Ruchala, K.J., et al. (2004) Megavoltage Computed Tomography Imaging: A Potential Tool to Guide and Improve the Delivery of Thoracic Radiation Therapy. Clinical Lung Cancer, 5, 303-306.

http://dx.doi.org/10.3816/CLC.2004.n.010

[15] Kubicek,. L.N., Seo, S., Chappell, R.J., et al. (2012) Helical Tomotherapy Setup Variations in Canine Nasal Tumor Patients Immobilized with A Bite Block. Veterinary Radiology Ultrasound, 53, 474-481. http://dx.doi.org/10.1111/j.1740-8261.2012.01947.x

[16] Jining, Z. (2009) Organ Motion and Image Guidance in Radiation. VCU Theses and Dissertations, Paper 1681.

[17] Murthy, V., Master, Z., Gupta, T., et al. (2010) Helical Tomotherapy for Head and Neck Squamous Cell Carcinoma: Dosimetric Comparison with Linear Accelerator-Based Step-and-Shoot IMRT. Journal of Cancer Research and Therapeutics, 6, 194-198. http://dx.doi.org/10.4103/0973-1482.65245

[18] Lian, J., Mackenzie, M. and Joseph, K. (2008) Assessment of Extended-Field Radiotherapy for Stage IIIC Endometrial Cancer Using Three-Dimensional Conformal Radiotherapy. Intensity-Modulated Radiotherapy and Helical Tomotherapy. International Journal of Radiation Oncology Biology Physics, 70, 935-943. http://dx.doi.org/10.1016/j.ijrobp.2007.10.021

[19] Mackie, T.R., Kapatoes, J., Ruchala, K., et al. (2003) Image Guidance for Precise Conformal Radiotherapy. International Journal of Radiation Oncology Biology Physics, 56, 89-105. http://dx.doi.org/10.1016/S0360-3016(03)00090-7

[20] Ramsey, C.R., Langen, K.M., Kupeian, P.A., et al. (2006) A Technique for Adaptive Image-Guided Helical Tomotherapy for Lung Cancer. International Journal of Radiation Oncology Biology Physics, 64, 1237-1244. http://dx.doi.org/10.1016/j.ijrobp.2005.11.012

[21] You, S.H., Seong, J., Lee, I.J., et al. (2008) Treatment Margin Assessment Using Mega-Voltage Computed Tomography of a Tomotherapy Unit in the Radiotherapy of a Liver Tumor. The Journal of the Korean Society for Therapeutic Radiology and Oncology, 26, 280-288. http://dx.doi.org/10.3857/jkstro.2008.26.4.280

[22] Mackie, T.R., Kapatoes, J., Ruchala, K., et al. (2003) Image Guidance for Precise Conformal Radiotherapy. International Journal of Radiation Oncology Biology Physics, 56, 89-105. http://dx.doi.org/10.1016/S0360-3016(03)00090-7

[23] Arumugam., S., Xing, A., Vial1, P., et al. (2013) Impact of Patient Rotational Errors on Target and Critical Structure Dose in IMRT: A 3D Simulation Study. The 7th International Conference on 3D Radiation Dosimetry (IC3DDose), 444, Article ID: 012045.

[24] Boswell, S.,Tome, W., Jeraj, R., et al. (2006) Automatic Registration of Megavoltage to Kilovoltage CT Images in Helical Tomotherapy: An Evaluation of the Setup Verification Process for the Special Case of a Rigid Head Phantom. Medical Physics, 33, 4395-4404. http://dx.doi.org/10.1118/1.2349698

[25] Bijhold, J., Lebesque, J.V. and Hart, A.A. (1992) Maximizing Setup Accuracy Using Portal Images as Applied to a Conformal Boost Technique for Prostatic Cancer. Radiotherapy Oncology, 24, 261-271. http://dx.doi.org/10.1016/0167-8140(92)90233-K

[26] Van Herk, M. (2004) Errors and Margins in Radiotherapy. Seminars in Radiation Oncology, 14, 52-64. http://dx.doi.org/10.1053/j.semradonc.2003.10.003

[27] The Royal College of Radiologist (2008) On Target: Ensuring Geometric Accuracy in Radiotherapy. The Royal College of Radiologist, London.

[28] Li, A. and Qi, S. (2007) Inter-Fractional Variations in Patient Setup and Anatomic Change Assessed by Daily Computed Tomography. International Journal of Radiation Oncology Biology Physics, 68, 581-591. http://dx.doi.org/10.1016/j.ijrobp.2006.12.024

[29] Booth, J.T. and Zavgorodni, S.F. (1999) Set-Up Error and Organ Motion Uncertainty: A Review. Australasian Physical \& Engineering Sciences in Medicine, 22, 29-47.

[30] Tuntipumiamorn, L., Liammookda, P. and Dechawongsuwan, S. (2013) Assessment of Inter-Fraction Setup Error of Non-Extended Standard Thermoplastic Mask for Head and Neck Intensity-Modulated Radiation Therapy Patients. Siriraj Medical Journal, 65, 31-35. 